\title{
Facilitated processing of visual stimuli associated with the body
}

\author{
Louise Whiteley \\ Department of Experimental Psychology, University of Oxford, South Parks Road, Oxford OX1 3UD, UK \\ Steffan Kennett, Marisa Taylor-Clarke, Patrick Haggard $\uparrow$ \\ Institute of Cognitive Neuroscience and Department of Psychology, University College London, \\ 17 Queen Square, London WC1N 3AR, UK; e-mail: p.haggard@ucl.ac.uk \\ Received 17 February 2003, in revised form 15 December 2003
}

\begin{abstract}
Recent work on tactile perception has revealed enhanced tactile acuity and speeded spatial-choice reaction times (RTs) when viewing the stimulated body site as opposed to viewing a neutral object. Here we examine whether this body-view enhancement effect extends to visual targets. Participants performed a speeded spatial discrimination between two lights attached either to their own left index finger or to a wooden finger-shaped object, making a simple distal-proximal decision. We filmed either the finger-mounted or the object-mounted lights in separate experimental blocks and the live scene was projected onto a screen in front of the participants. Thus, participants responded to identical visual targets varying only in their context: on the body or not. Results revealed a large performance advantage for the finger-mounted stimuli: reaction times were substantially reduced, while discrimination accuracy was unaffected. With this finding we address concerns associated with previous work on the processing of stimuli attributed to the self and extend the finding of a performance advantage for such stimuli to vision.
\end{abstract}

\section{Introduction}

The self is a fundamental concept of psychology. However, the possibility that the bodily self may be a special perceptual object has not been satisfactorily investigated. Previous experiments involving proprioception have not allowed comparison between processing of stimuli that are linked to the body and those that are not, because proprioception is a necessarily interoceptive modality. In addition, previous studies of peripersonal space in the visual modality (eg Hari and Jousmäki 1996) have failed to distinguish between stimuli linked to the body and stimuli located in the space around it. Therefore it remains unclear whether stimuli linked to the body are processed in the same way as other stimuli, or benefit from special perceptual processing. This special processing could contribute to the basis of self-consciousness.

Previous attempts to address this question directly have focused on interactions between vision and touch. For example, viewing a touched body site has been shown to improve perception of tactile stimuli (Kennett et al 2001; Tipper et al 1998). This effect is observed even when visual information is noninformative about the tactile stimulation. Kennett et al (2001) asked participants to make a two-point discrimination (2-PDT) judgment on the forearm. Prior to the tactile stimulation, participants viewed either their own arm or a neutral object appearing, via mirrors, in the same threedimensional location as their arm. The results showed increased tactile resolution (a lower 2-PDT) when the arm was viewed, even though no information about the stimulus was present in the view. Moreover, this effect remained even when spatial attention was controlled for.

These results are consistent with neurophysiological research on bimodal visualtactile neurons. These neurons are active whilst viewing the body directly (Graziano 1999), or indirectly (Iriki et al 2001), and during tool use (Iriki et al 1996). For example, 
Iriki et al (2001) trained monkeys to use a rake to reach food pellets on a table, and then trained them to solve this task by viewing their hand on a video monitor. After training, visual-tactile neurons that normally responded to visual stimuli near the hand extended their receptive field to incorporate the monitor. Behavioural findings in humans have shown similar results both in neuropsychological populations (Berti and Frassinetti 2000; Farnè and Làdavas 2000) and in healthy adults (Maravita et al 2002).

These results demonstrate an integration of visual and tactile representations when the body is part of the content of perception. This recalls the traditional notion of a 'body schema' (Head and Holmes 1911), defined as an abstract, higher-order representation of the position of the body parts in space - such a representation may supply top-down influences on unimodal perception. This process clearly matches visual and tactile inputs in quite a plastic way, since other objects attributed or linked to the body can be incorporated. For example, attribution of viewed objects, such as rubber hands, to the self has also been shown to alter tactile perception (Pavani et al 2000).

Other studies have demonstrated an interpersonal aspect to body representations. For example, visual judgments about the body parts of others are facilitated when participants move the corresponding part of their own body, compared with a condition where they move another body part (Reed and Farah 1995). These effects could also arise from an interaction between primary perceptual representations of the body and a higher-level abstract body schema.

When one touches something, one necessarily acquires information about one's own body: tactile receptors only encode 'bodily events', and in effect one 'perceives' the tactilereceptor surface. However, when one sees (or hears) something, one does not consciously receive information about the eyes/retinae or ears/basilar membranes, as the receptors of these exteroceptive modalities are not themselves part of the perception. Knowing whether similar modulatory effects occur in exteroceptive modalities such as vision is important to understanding the significance of the well-established tactile results. If perception is modulated when the body forms a constitutive part of the perceptual content (Bermúdez 2001), even in an exteroceptive modality, this would support the more general hypothesis that self-attributed stimuli undergo special processing. Thus, while the body is a physical object, stimuli linked to the body may receive special perceptual treatment. More speculatively, the activation of the special mode of processing for self-related stimuli could contribute to a neural substrate for self-consciousness. To address these issues, we investigated whether visual processing is modulated by viewing the body as opposed to a neutral object.

Experiments on visual processing of one's own body also avoid an important consequence of using touch in studies of self-representation. In previous tactile work attempts were made to control for confounds of attention across view conditions. In the experiment by Kennett et al (2001), participants viewed their own arm or a neutral object immediately prior to making a tactile judgment on the viewed arm. Both the arm and the object appeared to be in the same apparent three-dimensional location. Hence, spatial attention was controlled for between these conditions. However, when participants viewed the neutral object, they may have split their attention between the arm, on which they would receive the imperative stimulus, and the visible object. In contrast, when viewing the arm, only a single object was present, and hence a single focus for nonspatial object-based attention (Duncan 1984). In other words, the Kennett et al (2001) study controlled for spatial attention, but did not perfectly control for object-based attention. We directly controlled for both. In our experiment, participants reacted to visual targets on either their hand or a neutral object. Both were projected to the same spatial location, on a screen. Thus the view of the hand or the object was entirely incidental to the visual task and the number of objects demanding attention was constant across conditions. 
A previous experiment by Hari and Jousmäki (1996) used a similar design. When visual targets were projected onto the index fingers, RTs were shorter than when the visual targets were projected a small distance either side of the fingers. Slower reactions were observed when the separation of targets from fingers was achieved both by moving the target lights or by moving the responding fingers. However, this experiment differs from ours in an important way. In Hari and Jousmäki's study, subjects responded by pressing a key with the index finger closest to the visual target. Thus, the stimulus - response mapping varied across conditions, being most direct when the target was projected onto the responding finger itself, and less direct as the distance between target and responding digit was increased. Therefore, their experiment cannot distinguish between a response-based or a perception-based account. In contrast, our stimulus - response mapping was constant throughout.

Another consequence of the variation in stimulus-response mapping can be seen in the Hari and Jousmäki (1996) experiment, which failed to distinguish between a beneficial effect for stimuli linked to the body, and a beneficial effect for stimuli found in peripersonal space. Indeed, previous work has shown enhanced processing of stimuli appearing in peripersonal space (the space immediately surrounding the body; for a review see Làdavas 2002). The research discussed above, on visual-tactile neurons (eg Graziano and Gross 1992), also demonstrates special processing of peripersonal space and raises the question how this is defined. The receptive fields of these neurons are linked to parts of the body, but are flexible following tool use (eg Iriki et al 1996), and even incorporation of objects in extrapersonal space, such as video images (Iriki et al 2001). In the current experiment both the bodily self and the neutral object are similarly viewed outside a strictly defined peripersonal space-projected onto a screen. This dissociates possible effects of peripersonal space (defined as that immediately surrounding the body), from the bodily related nature of the stimulus. We suggest that activating an abstract representation of the body, or body schema, may produce topdown influences on perceptual processing. This effect might be independent of the spatial location of the stimulus. Our experimental hypothesis was therefore that processing of visual stimuli should be facilitated when these are viewed on the hand rather than on a neutral object.

\section{Methods}

\subsection{Participants}

Twelve paid volunteers (nine female, three male), aged 20 to 39 years (mean age $=26.9$ years) were studied. They reported normal or corrected-to-normal visual acuity. They were all naïve as to the purpose of the experiment. The participants had previously taken part in a temporal-order judgment task, judging which of two stimuli occurred first. One stimulus was a tactile buzz on the participant's right hand, and the other was an LED flash viewed on the left index finger or a neutral object.

\subsection{Apparatus}

The participant was seated, and placed her/his left forearm on a desk to the left of her/his midline, hand supine, with the index finger extended and resting on a fixed horizontal bar. A finger-sized block of wood served as the neutral object. This was placed on an identical bar elsewhere on the desk. There was a slim strap running the length of each horizontal bar, which was adjusted so as to hold the finger/fingershaped wooden block in place, and to minimise movement of the finger (see figure 1). This provided a similar visual background and reference frame across viewing conditions. Two identical pairs of LEDs were used, one pair attached to the finger and the other pair to the object, arranged along the axis of elongation of the digit/object. 


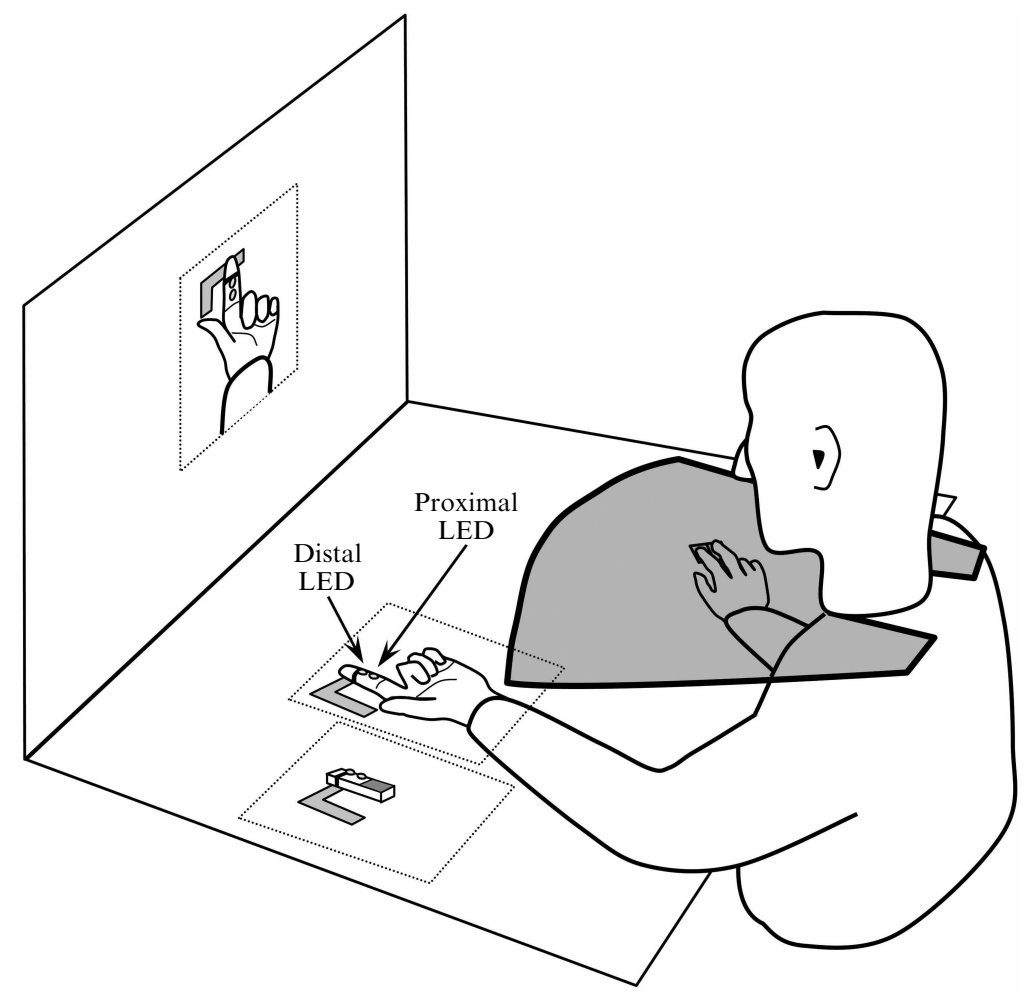

Figure 1. A schematic diagram showing the apparatus layout. Participants could not see any part of the table surface as they wore an occluding collar. They looked straight ahead at the image projected on the screen in front of them. This image was of the two target lights either mounted on their own left hand or the neutral object (dotted boxes approximate the boundaries of the two views which could be projected via a video camera). The finger and wooden block rested on identical horizontal bars with a slim strap across the middle of the finger or block holding it in position. This provided a similar reference frame for the distal-proximal decision in each case. The position, orientation, and luminance of the target lights was the same for the two projected images. The participant's right hand rested on two response keys.

Throughout the experiment the participant wore an occluding screen under her/his chin to prevent any direct view of the desk surface. Thus, no visual information as to the actual location of the finger or neutral object was available. A video camera was mounted on a tripod between the hand and object, pointing downwards, such that moving the camera through a small angle produced the two possible scenes, which were identical in size, shape, and orientation. These scenes were projected onto a screen, which was mounted $700 \mathrm{~mm}$ in front of the participant at eye level. Framing of the two scenes was controlled with a lens cover. The two scenes were lit with a lamp and care was taken to ensure the illumination and lighting were closely similar. The LEDs were positioned on the participant's finger $3 \mathrm{~mm}$ apart, and aligned along the distal-proximal axis (see figure 1). The proximal LED was positioned in the centre of the middle segment and the distal LED was placed at the boundary between the two distal segments. Identical LEDs were mounted at corresponding locations on the piece of wood. The angle of all LEDs was carefully adjusted to ensure that they pointed directly at the camera, and produced the same luminance for each participant and for both view conditions. This was monitored throughout the experiment.

The right hand was placed on the table, to the right of the participant's midline, near to two response keys. The index finger rested on one key and the middle finger on the other. 


\subsection{Procedure}

In each block, participants were instructed that they would view a pair of LEDs on either their own hand or the neutral object. Each trial began with a random foreperiod of 1100-2100 ms duration. Then a single LED flashed for $20 \mathrm{~ms}$. There followed $2020 \mathrm{~ms}$, during which participants made a speeded-choice response to identify which LED had flashed, pressing the index-finger key when the distal LED flashed and the middle-finger key when the proximal LED flashed. Thus there was a single stimulus response mapping between the LEDs and the response keys. Within a block each LED flashed on 10 trials, which were presented in a random order, making a total of 20 trials per block.

There were two view conditions, either the hand or the neutral object, with two blocks in each. The order of the view conditions was counterbalanced across participants.

\section{Results}

The participants' accuracy in pressing the correct response key was recorded as a percentage of trials. Participant median RTs were calculated from all correct trials, eliminating those few trials where the participant failed to make a response. The interparticipant mean of median RTs is shown in figure 2. Accuracy was identical in the two view conditions (hand: $98.0 \%$; object: $98.0 \%$ ), and the difference was not significant $\left(t_{11}=0.02\right.$, ns). However, RTs while viewing the hand were significantly shorter than while viewing the neutral object (584 ms versus $617 \mathrm{~ms} ; t_{11}=4.8, p<0.001$ ).

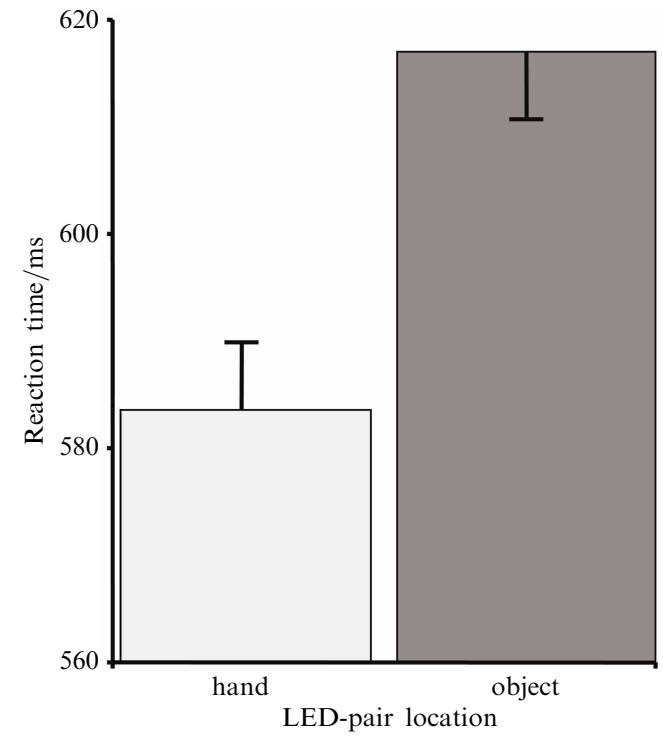

Figure 2. Mean RTs for the two view conditions. The bars show statistical significance bars (SSBs), relating to a one-tailed pairwise $t$-test. (For SSBs see C D Schunn, http:// www.Irdc.pitt.edu/schunn/SSB/.)

\section{Discussion}

We have examined the effect of viewing the hand on visual-choice RT. The clear finding was of faster responses when viewing the hand compared with viewing a neutral object. This speed advantage occurred in the absence of any effect on accuracy, suggesting a genuine performance improvement when the visual stimuli were mounted on the hand.

Our experiment removes some of the confounds in previous tactile and visual studies of self-representation, and again demonstrates that viewing the body can modulate perceptual performance. Previous studies have focused on the tactile modality, and could be open to alternative accounts based on spatial (Tipper et al 1998) or object-based attention (Kennett et al 2001). In this experiment we attempted to rule out such possible confounds, and also to isolate the source of the facilitated processing. 
First, the spatial location of the target stimuli was constant across conditions: their orientation, size, and ambient lighting was identical whether viewed next to the hand or the neutral object. Participants were naive as to the layout of the apparatus and had to direct their attention only to the target lights projected on the screen in front of them.

Second, object-based spatial attention was controlled for. Previous researchers have looked at tactile perception whilst participants viewed either the relevant body part or another object (eg Kennett et al 2001; Tipper et al 1998). This is open to the criticism that in one condition visual and tactile attention are on the same object, whereas in the other condition visual and tactile attention are focused on different objects. In the present study, only the visual modality was relevant, so there was no need to maintain attention on the hand when viewing the neutral object.

Third, since the visual targets were projected on a screen in front of participants, they never appeared within the immediate space of the body, termed peripersonal space (Làdavas 2002). Thus the enhanced performance for targets appearing near to the hand may be due to attribution of these stimuli to the body rather than simply due to their spatial location. This could be interpreted as showing that location in peripersonal space is not crucial for the body-related enhancement effect. However, our result could also motivate a redefinition of the term. We could define peripersonal space as space that is attributed to the body, rather than a stricter, physical definition as the space in close proximity to the body. According to this account, the stimuli on the projector screen occupy peripersonal space only when the projected scene is perceived as the participant's hand. This suggestion relates strongly to the work on bimodal visual-tactile cortical cells (eg Graziano and Gross 1992). These cells 'track' a particular body part across the visual and tactile modalities, and their receptive fields can be altered to incorporate tools (Iriki et al 1996) and views of the body outside strictly defined peripersonal space (Iriki et al 2001).

Our research also adds to the body of data suggesting that the process of attributing stimuli to the self is flexible in a number of interesting and important ways. For example, our result shows that stimuli not intrinsically experienced as part of the body (such as LEDs) can be attributed and processed as if they were part of the body. In future research, we plan to systematically manipulate the degree to which viewed body parts are attributed to the self in this visual-discrimination task.

We have hypothesised that the enhancement of processing when viewing the body may be related to the activation of a high-level body schema, which has a top-down effect on processing in single modalities. This may relate to more general work on top-down influences on unimodal processing (Driver and Spence 2000). In section 1 we suggested that to refine this hypothesis and to understand the functional significance of the tactile enhancement effect it is important to see whether such effects occur outside the tactile modality. Our result demonstrates that modulation of perception when the body is part of the perceptual content does extend to exteroceptive modalities. This provides tentative support to the idea that the body representation involved in the hypothesised top-down effect is amodal.

A further question is whether the generality of the effect just tells us something about the nature of the body schema involved, or whether it also tells us something about the nature of the individual sensory modalities. The traditional view is that touch and proprioception have a uniquely intimate and indubitable link to the self. As was discussed in section 1 , being touched seems to tell you something about the bodily self in an immediate way. However, our result could support the view that when processing the bodily self, there is greater commonality between sensory modalities than has traditionally been proposed. Indeed, recent research suggests both that (a) touch and proprioception are not perfect and indubitable sources of evidence about 
the body, and that (b) other modalities may also be flexible to top-down influence where the bodily self is concerned.

Intuitively, tactile perception does seem to be peculiarly immune to error-introspection tells us that we never need to ask the question "someone was just touched, but was it me?" However, other areas of research have suggested that tactile perception may not be as epistemologically privileged as this suggests. For example, Pavani et al (2000) showed that when tactile stimulation of a hand is combined with correlated visual information involving a rubber hand, a powerful illusion can take place. This involves attribution of the rubber hand to the self, and a corresponding mislocalisation of touch relative to the location of the rubber hand. Another example is the way in which the conscious perceptual content of touch may be dominated by either information about the skin surface, or by information about the external object. Thus, the brain has the ability to flexibly emphasise either interoceptive or exteroceptive touch. For example, when we use a tool to haptically explore an object, we seem to consciously perceive the contours of the object rather than our cutaneous sensations.

Our result supports the other side of the coin. Not only do touch and proprioception fail to live up to their indubitable reputation, but maybe other modalities are also flexibly influenced by the bodily content of perception.

As well as being relevant to recent empirical research, these ideas are also found in philosophical thought about self-consciousness, and about how we come to experience the world from a subjective perspective. For example, Brewer (1992) suggests that it is only through acting on the world and experiencing the multimodal sensory consequences that we come to separate our 'embodied selves' from the external world, and acquire a basic perceptual concept of the self that supports higher level self-consciousness. This idea is highly amenable to psychological research, via investigating perceptual and cognitive effects when the bodily content of perception is modulated. It is tempting to speculate that the present result supports the view that the activation of a special mode of processing for self-related stimuli could provide a neural substrate for selfconsciousness.

On a more methodological note, there are a number of future experiments that would strengthen the conclusion of this study, as well as helping us to make stronger inferences concerning the issues discussed above. One possibility that should be eliminated is that the enhanced processing of the visual stimuli on the hand reflects a general advantage for stimuli near any biologically significant objects, and we will investigate this in future studies.

Another open question, emphasised by one reviewer, is whether the modulation is an effect of self-attribution, or if it would appear also when viewing another person's hand. Debriefing of our participants revealed that they had experienced the image on the screen specifically as their own hand, and they were instructed at the beginning of the experiment that they would always view either their own left hand or an object. Our future research will systematically compare performance when participants view their own hands with that when viewing another person's hand. This will allow us to consider whether the abstract 'body schema' involved in the effect is interpersonal, or specific to the self.

In conclusion, our result seems to support the idea that self-attribution of stimuli activates an abstract body schema, which then has a top-down effect on unimodal processing. An experimental approach to perceptual processing of body-related stimuli may make the philosophical problems of self-consciousness more scientifically tractable.

Acknowledgments. This work was supported by grants from the Wellcome Trust to Louise Whiteley (VS/O2/UCL/24/LS/TH/FI), and the MRC to Steffan Kennett (G9627534). Patrick Haggard was supported by a Leverhulme Trust Research Fellowship and Marisa Taylor-Clarke was supported by the EPSRC. 


\section{References}

Bermúdez J L, 2001 "Nonconceptual self-consciousness and cognitive science" Synthese 129129 - 149

Berti A, Frassinetti F, 2000 "When far becomes near: Remapping of space by tool use" Journal of Cognitive Neuroscience $12415-420$

Brewer B, 1992 "Self-location and agency" Mind 101 17-34

Driver J, Spence C, 2000 "Multisensory perception: Beyond modularity and convergence" Current Biology 10 R731 - R735

Duncan J, 1984 "Selective attention and the organization of visual information" Journal of Experimental Psychology: General $113501-517$

Farnè A, Làdavas E, 2000 "Dynamic size-change of hand peripersonal space following tool use" Neuroreport $111645-1649$

Graziano M S A, 1999 "Where is my arm? The relative role of vision and proprioception in the neuronal representation of limb position" Proceedings of the National Academy of Sciences of the United States of America $9610418-10421$

Graziano M S A, Gross C G, 1992 "Somatotopically organized maps of near visual space exist" Behavioral and Brain Sciences 15750

Hari R, Jousmäki V, 1996 "Preference of personal to extrapersonal space in a visuomotor task" Journal of Cognitive Neuroscience $8305-307$

Head H, Holmes G, 1911 "Sensory disturbances from cerebral lesions" Brain $34102-254$

Iriki A, Tanaka M, Iwamura Y, 1996 "Coding of modified body schema during tool use by macaque postcentral neurones" Neuroreport $72325-2330$

Iriki A, Tanaka M, Obayashi S, Iwamura Y, 2001 "Self-images in the video monitor coded by monkey intraparietal neurons" Neuroscience Research 40163 - 173

Kennett S, Taylor-Clarke M, Haggard P, 2001 "Noninformative vision improves the spatial resolution of touch in humans" Current Biology 11 1188-1191

Làdavas E, 2002 "Functional and dynamic properties of visual peripersonal space" Trends in Cognitive Sciences 6 17-22

Maravita A, Spence C, Kennett S, Driver J, 2002 "Tool-use changes multimodal spatial interactions between vision and touch in normal humans" Cognition 83 B25-B34

Pavani F, Spence C, Driver J, 2000 "Visual capture of touch: Out-of-the-body experiences with rubber gloves" Psychological Science 11353 - 359

Reed C L, Farah M J, 1995 "The psychological reality of the body schema: A test with normal participants" Journal of Experimental Psychology: Human Perception and Performance 21 $334-343$

Tipper S P, Lloyd D, Shorland B, Dancer C, Howard L A, McGlone F, 1998 "Vision influences tactile perception without proprioceptive orienting" Neuroreport $91741-1744$ 


\section{PERCEPTION}

vOLUME 332004

www.perceptionweb.com

Conditions of use. This article may be downloaded from the Perception website for personal research by members of subscribing organisations. Authors are entitled to distribute their own article (in printed form or by e-mail) to up to 50 people. This PDF may not be placed on any website (or other online distribution system) without permission of the publisher. 